\title{
Low-frequency detection and discrimination following apical hair cell destruction
}

\author{
Cynthia A. Prosen * and David B. Moody \\ Kresge Hearing Research Institute, The University of Michigan Medical School, Ann Arhor, Michigan, U.S.A.
}

(Received 5 November 1990; accepted 11 August 1991)

\begin{abstract}
This study assessed the effects of apical hair cell destruction on the detection and discrimination of low-frequency stimuli. Monauralized chinchillas were trained using operant conditioning and positive reinforcement to respond to pure-tone stimuli in the absence and the presence of a high-pass noise masker. Following the collection of the baseline absolute thresholds, psychophysical tuning curves (PTCs) also were determined at low and high frequencies. Apical hair cells in the experimental ear of each subject then were destroyed by applying a liquid-nitrogen-cooled miniature cryoprobe to the bony wall of the cochlea. Post-cryosurgery, unmasked and masked absolute thresholds and psychophysical tuning curves were re-evaluated. Following cryosurgery, low-frequency absolute thresholds increased by $30.50 \mathrm{~dB}$. High pass masking data suggested that receptors that were unaffected by the masking noise were responsible for the remaining low-frequency hearing. Low-frequency tuning, monitored by assessing changes in PTCs, was significantly altered following apical receptor cell loss, with the most effective maskers located several octaves above the test tone frequency. Following these determinations, one control and two experimental subjects then participated in a third experiment assessing low-frequency discrimination acuity. Some discrimination ability was retained after the cryosurgery: however. these post-lesion difference limens increased when a high-pass noise masker was added to the test environment. At the termination of the behavioral experiment, subjects were euthanized and their cochleae dissected to correlate behavioral and histopathological data. The data suggest that receptors located in frequency regions of the cochlea normally responsive to middle and high frequencies may be responsible for detection and discrimination of low-frequency stimuli in apically damaged cochleae. These data are consistent with other reports which indicate that redundant mechanisms are available for the detection of low-frequency stimuli, and they provide new information regarding how low-frequency stimuli are discriminated throughout the cochlea.
\end{abstract}

Cochlear apex; Low-frequency hearing; Frequency discrimination: Frequency selectivity; High-pass masking

\section{Introduction}

Behavioral and histopathological data suggest that there are redundant coding mechanisms in the mammalian cochlea for low-frequency signals, as reviewed by Prosen et al. (1990b). Specifically, following significant but restricted destruction of apical receptor cells, behaviorally-assessed thresholds for low-frequency pure tones increased by $10-20 \mathrm{~dB}$ in guinea pigs and chinchillas. Only when both basal and apical hair cells were destroyed did thresholds for low-frequency signals shift more than $20 \mathrm{~dB}$. These data, together with the results of a high-pass noise-masking experiment, indicated that in the complete absence of those apical receptor cells normally tuned to low-frequency signals, undamaged mid- and basal-turn cells responded to low frequencies. Prosen et al. (1990b) suggested that an important ques-

\footnotetext{
* Present address: Department of Psychology, Northern Michigan University, Marquette, MI 49855, U.S.A.

Correspondence to: David B. Moody, Kresge Hearing Research Institute. University of Michigan Medical School. Ann Arbor, MI 481090506, U.S.A. Fax: (313) 764-0014.
}

tion to explore in an apically-damaged ear is the extent to which the remaining functional receptor cells, which can be used to detect low-frequency signals, are useful in discriminating between suprathreshold low-frequency stimuli.

Changes in frequency selectivity, assessed with either forward- or simultaneous-masked psychophysical tuning curves (PTCs) in listeners with high-frequency sensorineural hearing loss, have been described in the literature, with most investigators concluding that high-frequency PTCs are broadened in those listeners, as reviewed by Smith et al. (1987c). While reports of changes in frequency selectivity in listeners with a predominant low-frequency hearing loss are much less common, those studies that have been conducted concur that frequency selectivity is altered (Thornton and Abbas, 1980; Goldstein et al., 1983; Long and Cullen, 1988; Florentine and Houtsma, 1983). These studies report that PTCs are abnormally tuned, with the maximum masking frequency much higher than the frequency of the test tone, suggesting that the detection of the test tone occurred at a basal cochlear location. Because these studies were conducted with human 
listeners, no histological data were available to correlate the altered psychophysical abilities with a defined pattern of cochlear destruction.

The results of studies exploring the ability of listeners with low-frequency hearing loss to tell the difference between successively presented discrete stimuli that differ only in frequency are more ambiguous than those describing frequency selectivity in this kind of listener. Because cells throughout the cochlea may be capable of encoding low frequencies, Butler and Albrite (1956) suggested that low-frequency discrimination ability should be less affected by selective apical-cell loss than high-frequency discrimination ability following selective basal-cell loss. Alternatively, others have suggested that, for a given degree of hearing loss, deterioration in frequency discrimination ability is greater for low-frequency tones than for high-frequency tones (Turner and Nelson, 1982; Zurek and Formby, 1981; Prosen et al., 1989a, 1989b). Clark and Bohne (1986), for example, reported that low-frequency discrimination in a chinchilla with partial apical receptor cell destruction was disrupted when the low-frequency absolute threshold shift was less than $30 \mathrm{~dB}$. One explanation for these disparate data may be that frequency discrimination at low frequencies can be mediated by both tonotopic and temporal processes. Turner et al. (1983) and Florentine and Houtsma (1983) suggested that while low-frequency discrimination is normally conducted tonotopically, the process may be mediated temporally in low-frequency sensorineural hearing loss listeners. Psychophysical data suggest that different frequencies of temporal stimulation can produce distinct pitch-like sensations from one place in the cochlea, as noted by Turner et al. (1983).

The present experiments were designed to assess the contribution of the remaining functional cells to low-frequency tuning and low-frequency discrimination ability in chinchillas with reduced populations of apical receptor cells.

\section{Methods}

\section{Subjects}

Subjects were 5 male chinchillas (Chinchilla lanigera) obtained as fur culls from a local breeder. All were less than one year of age at the start of the experiment. Incoming animals were screened for normal hearing using the auditory brainstem response (ABR), and only animals which exhibited ABR thresholds within normal limits were used as behavioral subjects. Prior to experimentation, the subjects were gradually reduced to 80 $90 \%$ of their free-feeding body weight by restricting their daily food ration. Details of maintenance feeding and housing are provided in Prosen et al. (1990b).
All animals were surgically monauralized prior to behavioral training by disarticulation of the right ossicular chain and cryoprobe destruction of the entirc right cochlea. The four experimental subjects had the cryoprobe applied to their left apical cochlea, while the control subject $(\mathrm{C} 125)$ provided normal frequency discrimination data, as described below.

\section{Apparatus}

Behavioral testing was conducted inside a doublewalled, sound-attenuating chamber (Industrial Acoustics). The testing cage was constructed of hardware cloth, and was located in the middle of the chamber. A cue light, response lever, and pellet delivery tray were located on one wall of the cage. A high-frequency speaker (Motorola piezoelectric model 71905A) was mounted directly above the response lever, and a lowfrequency speaker (Audio Dynamics XT'-6 with the tweeter disconnected) was suspended from the ceiling of the room such that it also hung directly over the response lever when the high-frequency speaker was removed. During the masking experiments, the masker was delivered through a second Audio-Dynamics XT-6 speaker (with the tweeter functional). The masking speaker was mounted either directly in front (for PTC tests) or to the left (for $\Delta \mathrm{F}$ tests) of the testing cage with the axis of the speaker at the level of the subject's head.

\section{Stimuli}

Acoustic test stimuli were generated either by lowdistortion oscillators (Krohn-Hite 4500 or $4030 \mathrm{R}$, HP 200CDR) or by digital synthesis techniques. Oscillatorgenerated stimuli were used for absolute threshold and PTC determinations. Digitally synthesized stimuli were used for the $\Delta \mathrm{F}$ testing and were generated on an AT-class computer using the 12-bit D/A converters of a Data Translation DT2821F analog subsystem. The digitized waveforms were output at a $20-\mathrm{kHz}$ clock rate, and the output of the D/A converter was low-pass filtered through both halves of a Krohn-Hite variable filter (model 3343 ) set to a $4-\mathrm{kHz}$ cutoff, low-pass, max-flat mode. The computer stimuli were generated with 20-ms Gaussian rise/fall times; the oscillator stimuli were gated through tone switches, also with 20 -ms rise/fall times. In all but one testing situation, stimuli from 0.063 to $2.0 \mathrm{kHz}$ were presented through the low-frequency speaker, while stimuli above $2.0 \mathrm{kHz}$ were presented through the high-frequency speaker. In the post-lesion, low-frequency PTC testing situation, the test tones and all maskers $(0.063-8.0 \mathrm{kHz})$ were presented through the low-frequency speaker.

The high-pass masking noise was generated by a General Radio 1381 noise generator, high-pass filtered by an Allison $2 \mathrm{ABR}$ variable filter set to produce a 
3-dB down point at $0.71 \mathrm{kHz}$, and then passed through an equalizer network (Altec model 8960A). The rolloff of this combination was approximately $45 \mathrm{~dB}$ /octave. The masker had a flat spectrum up to $12 \mathrm{kHz}$ and showed approximately a $10-\mathrm{dB}$ decrease from 12 to $22.4 \mathrm{kHz}$.

Free-field sound calibrations were carried out using Bruel and Kjaęr 1/2- and 1/4-in microphones. Microphone output was measured at 5-7 locations in the test cage, sampling positions likely to be occupied by the test ear of the subject when the key was depressed. Sound pressures measured at each of these locations were averaged to produce a representative calibration value at each frequency. The masking noise spectrum was calibrated using the 1/2-in microphone and a wave analyzer (Hewlett-Packard model 3590a). Overall masker levels were measured with a sound-level meter using the $\mathrm{B}$ weighting.

\section{Procedure}

\section{Training and absolute threshold determination}

Subjects were trained to press the response lever when the flashing cue light was on, and to release it whenever a 2.5-s auditory stimulus was presented. Details of the training procedure are provided by Smith et al. (1987a). The psychophysical method of tracking was used to determine absolute thresholds. Data were considered stable when 4 of 5 consecutive daily thresholds at each test frequency were within $10 \mathrm{~dB}$.

\section{High-pass masking}

Following determination of stable baseline thresholds at octave and half-octave frequencies throughout the range of sensitivity, masked thresholds were determined in the presence of a high-pass noise masker having a cutoff at $0.71 \mathrm{kHz}$. The purpose of this masker was to reduce the contribution of receptors from highfrequency regions to hearing. The masker level was increased until a threshold shift in the presence of the masker was observed at frequencies below the $0.71-\mathrm{kHz}$ cutoff. After a complete threshold shift function was determined at that masker level, the level was decreased by $10 \mathrm{~dB}$, and a second threshold shift function was determined. It was assumed that this lower level approximated the maximum possible level that would not directly mask low-frequency regions of the auditory system. These levels were individually determined for each subject and ranged from 42 to $53 \mathrm{~dB}(\mathrm{~B})$.

\section{Baseline psychophysical tuning curves}

Baseline PTCs were obtained at 0.355 and $8.0 \mathrm{kHz}$ from each subject prior to cryolesioning, using a simultaneous masking procedure. Although simultaneous masking can result in irregularities in the PTC near the frequency of the test tone due to beating between the test tone and the masker, this procedure was used in the present experiment because a) it was similar to that used to determine thresholds in the presence of the high-pass masker, requiring little additional training time, and $b$ ) the focus of the experiment was to monitor shifts in the minima of the PTC functions following apical hair cell damage, rather than to define the exact shape of the tip of the tuning curve. PTCs were determined with the test tone at $10 \mathrm{~dB}$ SL unless a subject failed to produce stable data at that level, in which case a $20 \mathrm{~dB}$ SL level was used. Masker frequencies bracketed the test-tone frequency and encompassed both tails of the P'IC:

When the subject initiated the lever-holding response, a pure-tone masker was presented and remained on as long as the lever was depressed. As in the absolute threshold testing procedure, the test tonc was presented after the lever had been depressed for a variable amount of time. Correct detections of the tone resulted in delivery of a food pellet and a $10-\mathrm{dB}$ increase in the level of the masker on the next trial. Failure to detect the test tone resulted in a $10-\mathrm{dB}$ decrease in masker level. Tuning curves were derived by determining the level of the masker at each frequency that resulted in detection of the test tone on $50 \%$ of the trials, and were considered stable when values at each masker frequency fell within a $10-\mathrm{dB}$ range $( \pm 5 \mathrm{~dB}$ of the median value) for 3 consecutive days.

\section{Cryosurgery}

The surgical procedure used for producing the cryolesions has been described in detail (Brown and Nuttall, 1987). Briefly, under sterile conditions, the bulla was opened and the tip of a liquid-nitrogen-cooled cryoprobe was applied to the apical turn of the cochlea for $1.5 \mathrm{~min}$. For 2 of the 4 subjects (C128 and C122), the cryoprobe was then removed and re-cooled for 3 min, and again applied for $1.5 \mathrm{~min}$. For $\mathrm{C} 134$, two 1-min cryoprobe applications were employed, with a 2-min inter-application interval. The fourth subject, C130, had an irregular schedule of cryoprobe applications and removals $(53 \mathrm{~s}$ on, $27 \mathrm{~s}$ off, $25 \mathrm{~s}$ on, $3 \mathrm{~min}$ off, $1 \mathrm{~min} 18 \mathrm{~s}$ on, $4 \mathrm{~min}$ off, $30 \mathrm{~s}$ on; for a total of $3 \mathrm{~min}$ and $6 \mathrm{~s}$ on). This schedule was used because $\mathrm{C} 130 \mathrm{had}$ an unusual nerve placement over the bulla that precluded normal access to the cochlear apex, resulting in difficulty in holding the cryoprobe tip in position for the desired period of time. For all subjects, the bulla was not closed after cryolesioning; however, unpublished data from our laboratory indicate that healing occurs within 6-8 weeks. No prophylactic antibiotics were given post surgery. Behavioral testing of all experimental subjects resumed within 1-2 days of surgery.

\section{Post-lesion testing}

Following cryosurgery, absolute threshold testing continued until the hearing loss stabilized. Thresholds 
then were redetermined in the presence of that level of the high-pass masking noise which did not produce any threshold shift below $0.71 \mathrm{kHz}$ during baseline determinations. Following those absolute threshold measurements, PTCs were redetermined at 0.355 and 8.0 $\mathrm{kHz}$. For $\mathrm{C} 134$, the post-lesion PTC testing was carried out with a $0.71-\mathrm{kHz}$ test tone to avoid masker levels above $80 \mathrm{~dB}$ SPL that might produce additional hearing loss. As in the baseline PTC determinations, testing was carried out at 10 or $20 \mathrm{~dB}$ SL.

\section{Post-lesion pure-tone frequency discrimination}

Because the post-lesion threshold shift functions remained stable throughout the time period required to redetermine PTCs for 3 of the 4 experimental subjects, the original experimental protocol was modified to measure post-lesion $\Delta \mathrm{Fs}$ at $355 \mathrm{~Hz}$ from two of the subjects, C128 and C134, thereby obtaining two measures of discriminability from the lesioned animals. Since this protocol modification occurred after the lesions were produced, baseline values from $\mathrm{C} 128$ and $\mathrm{C} 134$ were not obtained; rather, $\Delta \mathrm{F}$ comparisons were made to published values, as discussed below, and to data from $\mathrm{C} 125$, the control subject tested with the same procedure. Although such between-subject comparisons are inherently less powerful than within-subject comparisons, as was done in the PTC portion of the experiment, the availability of the trained subjects with extensive post-lesion data provided a unique opportunity to obtain multiple measures of hearing and correlative histopathological data from the same animals.

Full details of the procedure for measuring difference thresholds are presented in Prosen et al. (1990a). Stimuli were presented according to the psychophysical method of constant stimuli. The level of the standard tone pulses was randomized within $\pm 5 \mathrm{~dB}$ of the 10-dB SL testing level. Levels for each of the comparison stimuli were set to $10 \mathrm{~dB}$ SL based on absolute threshold determinations conducted once per weck at each comparison frequency. Threshold was defined as the frequency difference that yielded $50 \%$ correct reports. The range of comparison stimuli was selected to include 1 or 2 values yielding less than $50 \%$ correct. The criterion used to define stability required 4 of 5 successive determinations to be within $\pm 10 \%$ of the median threshold for the 5 determinations. Following $\Delta \mathrm{F}$ measurement in silence, the high-pass masker was introduced, and the $355-\mathrm{Hz} \Delta \mathrm{F}$ threshold was redetermined. Finally the masker was removed, and the unmasked $\Delta \mathrm{F}$ threshold assessed again.

\section{Histological evaluation}

When all behavioral data had been collected, subjects were heavily anesthetized with chloral hydrate $(17.5 \%, 0.2 \mathrm{ml} / 100 \mathrm{~g})$ and perfused through the heart with fixative containing $2.5 \%$ glutaraldehyde and $2 \%$ paraformaldehyde in $0.1 \mathrm{M}$ sodium cacodylate buffer, pH 7.3. After 10 minutes of fixation the animals were decapitated and the temporal bones removed. The bullae were exposed and the middle ears were examined for possible infection. Round and oval windows then were exposed and opened and the same fixative was gently perfused through the round window. After 1 $h$ in fixative, the cochleae were rinsed in buffer. This was followed by intrascalar perfusion of $1 \% \mathrm{OsO}_{4}$ and a second series of rinses. The otic capsule (bony shell) then was removed and stria and segments of the cochlear spiral removed to obtain surface preparations. Segments were usually half to complete cochlear turns, except in cases of severe damage. These segments were mounted on slides under phosphate-buffered saline and glycerin $(1: 3)$, coverslipped and examined on a Leitz photomicroscope under phase-contrast or differential-interference-contrast optics. The number of missing hair cells was assessed in all segments of the entire cochlear spiral. These data were used to construct cytocochleograms in which the percentage of inner and outer hair cells was plotted as a function of percent distance from the apex of the cochlea. Cells were counted as present if either the stereocilia or the cell nucleus could be visualized. No attempt was made to assess possible cellular damage to the surviving cells.

\section{Results}

\section{Baseline data}

The baseline absolute thresholds for the four experimental animals and the control subject were within a normal range, as defined by Prosen et al. (1989a). For 3 of the 4 experimental animals, the post-lesion masking data described below were collected at levels at which, in the pre-lesion testing situation, there was a threshold shift in the presence of the masker at frequencies $1.0 \mathrm{kHz}$ and above, but no shift at frequencies $0.71 \mathrm{kHz}$ and below. For the fourth subject, post-lesion masking data were collected at the 2 levels used pre-lesion; at the higher level, there was a 5-10 dB threshold shift at frequencies $0.71 \mathrm{kHz}$ and below in the pre-lesion masked testing situation. For all 4 subjects, the low-level pre-lesion masker was ineffective in shifting thresholds at $32.0 \mathrm{kHz}$, probably because the spectrum of the masker was not flat out to high frequencies.

Hearing loss, cytocochleograms, and post-lesion masking data

The upper halves of Figs. 1, 2 and 3 show cytocochleograms from the experimental ears of $\mathrm{C} 122$, $\mathrm{C} 130$ and $\mathrm{C} 134$, respectively, while the lower halves 


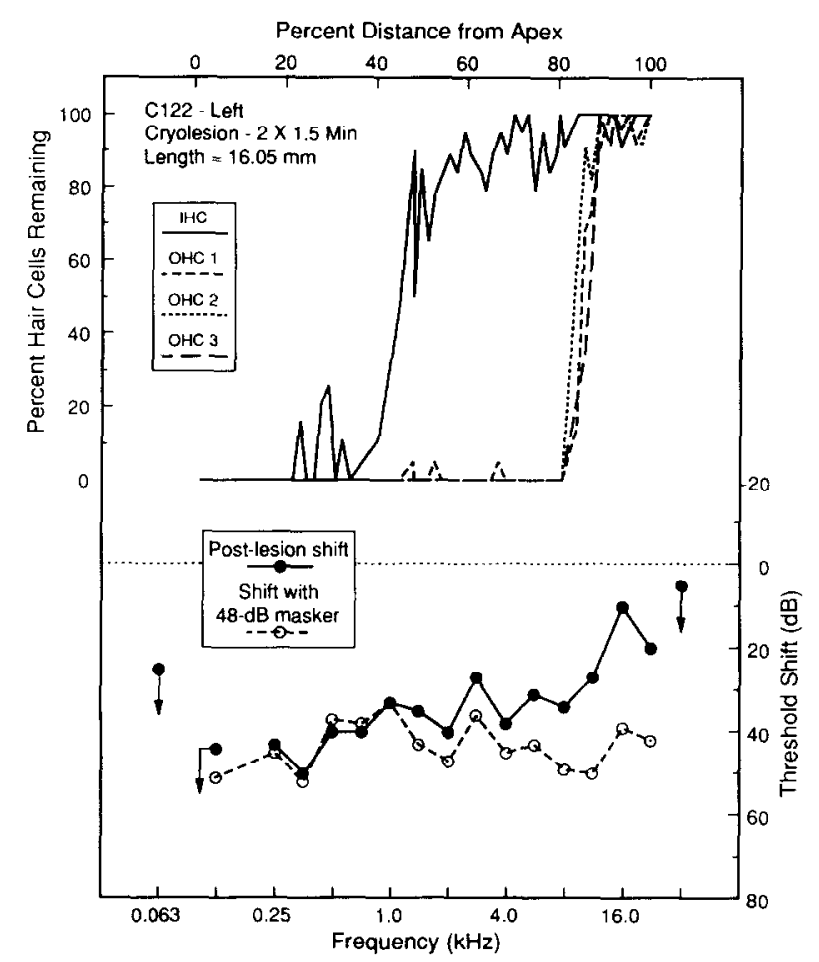

Fig. 1. The upper half of this figure depicts the percentages of inner and outer hair cells remaining as a function of the relative distance from the apex of the cochlea from the experimental ear of C122. This subject had the tip of the cryoprobe placed on the apex of the bony wall of the cochlea for 2 applications of $1.5 \mathrm{~min}$ each, with an inter-application interval of $3 \mathrm{~min}$. The lower half of this figure shows the extent of C122's hearing loss after cryosurgery, both in the absence (closed circles) and the presence (open circles) of a high-pass wide-band noise masker $(48 \mathrm{~dB}(\mathrm{~B}))$ with a low-frequency cutoff at $0.71 \mathrm{kHz}$. Points with downward-pointing arrows represent frequencies where thresholds could not be obtained because equipment limitations prevented presenting higher sound levels. Frequency and distance axes are aligned according to the method of Eldredge et al. (1981).

show hearing loss with and without the high-pass masker. Data from C122 (Fig. 1) suggest that in the absence of nearly all receptor cells in the apical $40 \%$ of the cochlea, absolute thresholds at frequencies $1.0 \mathrm{kHz}$ and below increased $30-50 \mathrm{~dB}$. Thresholds from 1.4$11.2 \mathrm{kHz}$, which increased by $30-40 \mathrm{~dB}$, were correlated with complete outer hair cell loss and 70-90\% inner hair cell retention. Finally, the $10-20 \mathrm{~dB}$ hearing loss from $16.0-32.0 \mathrm{kHz}$ was correlated with retention of all inner and nearly all outer hair cells.

Iwo permanent threshold shift functions are displayed for C130 (Fig. 2). Data for the post-lesion shift function were collected 2-3 weeks after cryosurgery. These data indicate that frequencies $0.71 \mathrm{kHz}$ and below shifted $20-40 \mathrm{~dB}$, while thresholds at higher frequencies were elevated $0-15 \mathrm{~dB}$. Correlated with this hearing loss function, partial inner and outer hair cell loss was noted in the apical $60 \%$ of the cochlea, while hair cells in the remainder of the cochlea were present. Hair cells in a part of C130's apical cochlea could not be counted because the tissue there was severely damaged. Such severe damage was frequently noted in the cryolesioned ears, making dissection and histological evaluation difficult.

During the period when C130's post-lesion PTCs were assessed, absolute thresholds at all frequencies became elevated by an additional $20-30 \mathrm{~dB}$, as indicated by the 'pre-sacrifice shift' function. The magnitude of this shift is similar to that reported in humans with otitis media (Klein, 1983). Concurrent with this threshold elevation, $\mathrm{C} 130$ developed a severe right eye infection, while otoscopic inspection suggested that both the right and the left middle ear spaces were infected. C130's behavior began to deteriorate shortly after the masking data and the $8.0 \mathrm{kHz}$ post-lesion PTC data described below were collected. Neither the eye nor the ear infections responded to aggressive non-ototoxic antibiotic therapy, and the decision was

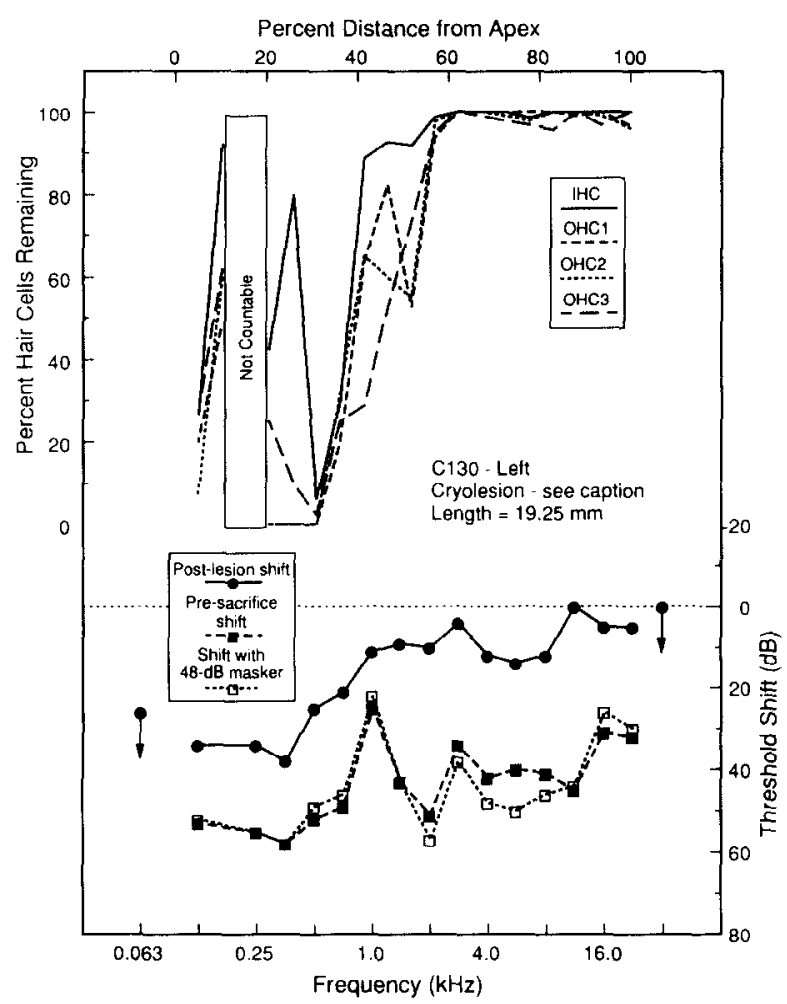

Fig. 2. The upper half of this figure depicts the percentages of inner and outer hair cells remaining as a function of the relative distance from the apex of the cochlea from the experimental ear of C130. This subject had the tip of the cryoprobe placed on and removed from the apex of the bony wall of the cochlea according to the following schedule: $53 \mathrm{~s}$ on, $27 \mathrm{~s}$ off, $25 \mathrm{~s}$ on, $3 \mathrm{~min}$ off, $1 \mathrm{~min} 18 \mathrm{~s}$ on, $4 \mathrm{~min}$ off, $30 \mathrm{~s}$ on. The lower half of this figure shows the extent of C130's hearing loss assessed 2-3 weeks after cryosurgery (closed circles), and shortly before sacrifice in the absence (closed squares) and the presence (open squares) of a high-pass wide-band noise masker $(48 \mathrm{~dB})$ with a low-frequency cutoff at $0.71 \mathrm{kHz}$. Points with downward-pointing arrows represent trequencies where thresholds could not be obtained hecasse cquipment limitations prevented presenting higher sound levels. Frequency and distance axes are aligned according to the method of Eldredge et al. (1981). 


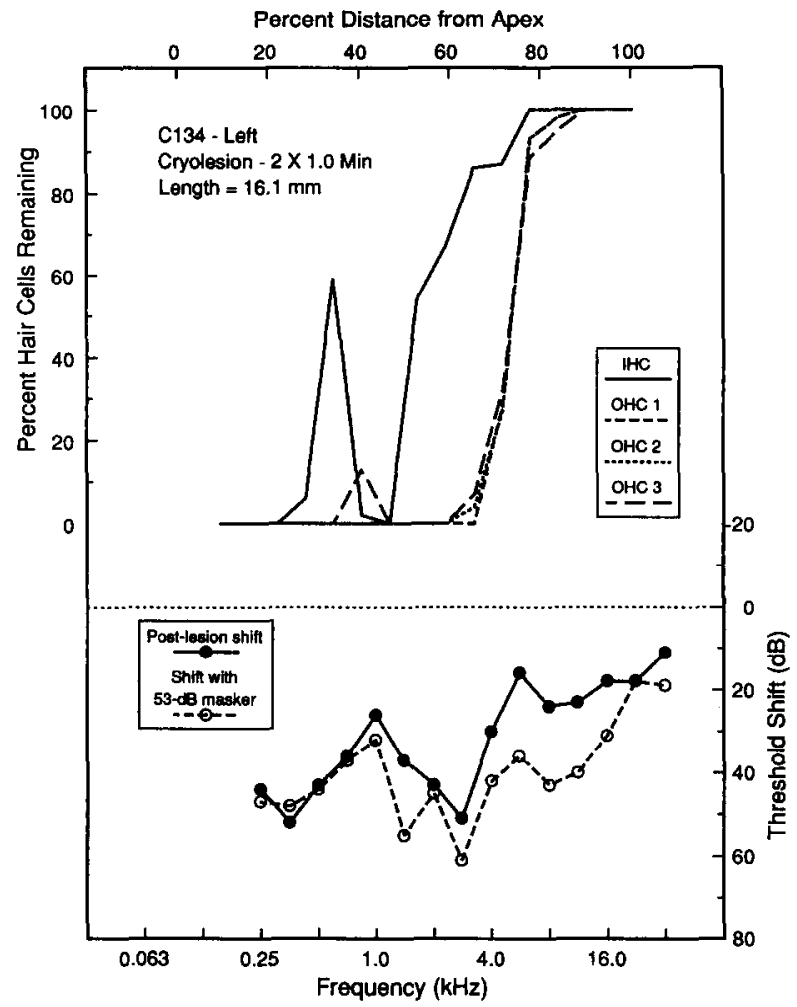

Fig. 3. The upper half of this figure depicts the percentages of inner and outer hair cells remaining as a function of the relative distance from the apex of the cochlea from the experimental ear of C134. This subject had the tip of the cryoprobe placed on the apex of the bony wall of the cochlea for 2 applications of $1 \mathrm{~min}$ each, with an inter-application interval of $2 \mathrm{~min}$. The lower half of this figure shows the extent of C134's hearing loss after cryosurgery, both in the absence (closed circles) and the presence (open circles) of a high-pass wide-band noise masker (53 $\mathrm{dB}(\mathrm{B}))$ with a low-frequency cutoff of $0.71 \mathrm{kHz}$. Frequency and distance axes are aligned according to the method of Eldredge et al. (1981).

made to sacrifice this animal rather than risk losing the opportunity to evaluate the histopathology from the left ear. Sacrifice occurred before the $0.355 \mathrm{kHz}$ postlesion PTC data were obtained. Histological examination of the left middle ear space of C130 indicated a purulent middle ear infection. The presence of the middle ear infection suggested that the histological data are more appropriately correlated with the absolute threshold shift function determined immediately post-lesion.

The absolute thresholds of $\mathrm{C} 134$ (Fig. 3) were elevated by $30-50 \mathrm{~dB}$ at frequencies $4.0 \mathrm{kHz}$ and below, while thresholds at higher frequencies increased by 10-20 dB. Corresponding to these data, nearly all of the outer hair cells and most of the inner hair cells werc absent in the apical $45 \%$ of the cochlea. The more moderate high-frequency threshold shifts were correlated with gradually increasing numbers of outer hair cells, and good inner hair cell retention.

Fig. 4 depicts the hearing loss of $\mathrm{C} 128$ post-cryosurgery. Thresholds shifted from $40-60 \mathrm{~dB}$ at frequen- cies below $2.0 \mathrm{kHz}$, from $20-40 \mathrm{~dB}$ at frequencies 2.8 , 4.0 , and $11.2-22.4 \mathrm{kHz}$, and from $0-5 \mathrm{~dB}$ at 5.6 and 8.0 kIIz. Dissection of C128's experimental ear indicated that both inner and outer hair cells were present in the hook region of this animal's cochlea, but the remaining tissue was inadequately stained and in such poor condition that an accurate cell count was precluded, making a correlation between hearing loss and hair cell loss impossible.

Post-lesion masking data from all four subjects indicate that in the presence of the high-pass masker, thresholds at high frequencies increased, while thresholds at the lower frequencies were unchanged from their post-lesion, unmasked values. Data in Fig. 4 indicate that even when the higher level of masker was used, which increased low-frequency thresholds in the pre-lesion testing situation, low-frequency thresholds were not elevated. These data suggest that the lowfrequency stimuli were encoded by receptor cells whose characteristic frequency was not within the masking band, or alternatively, as discussed below, by receptors tuned to frequencies within the masking band that were not themselves affected by the masker.

\section{Pre- and post-lesion psychophysical tuning curves}

Pre- and post-lesion PTCs for the 4 experimental subjects are seen in Figs. 5-8. The histopathology was similar for $\mathrm{C} 122$ and $\mathrm{C} 134$ from the region of the basilar membrane that is maximally responsive to 0.355 $\mathrm{kHz}$ in undamaged cochleae. That is, both of these subjects had only a small percentage of inner hair cells

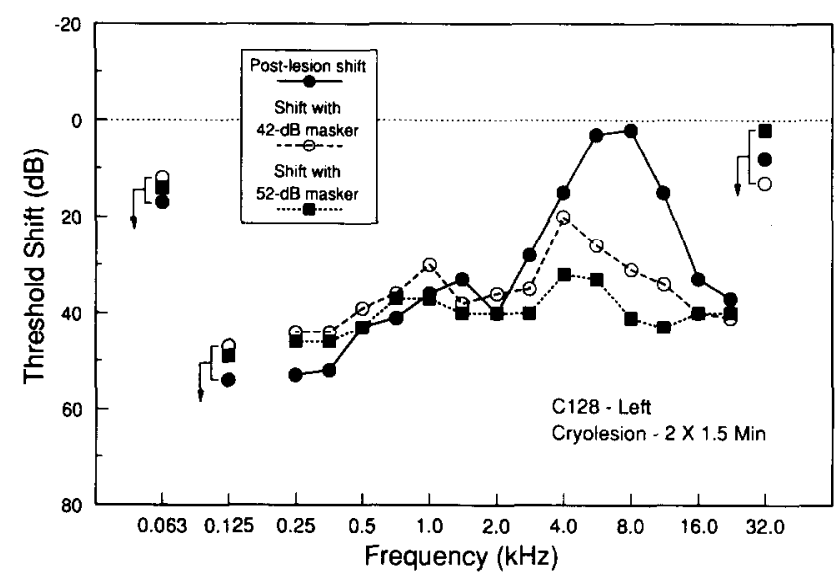

Fig. 4. This figure shows the extent of C128's hearing loss after cryosurgery, in the absence (closed circles) and the presence of a 42 $\mathrm{dB}$ level (open circles) or a $52 \mathrm{~dB}(\mathrm{~B})$ level (closed squares) high-pass wide-band noise masker with a low-frequency cutoff of $0.71 \mathrm{kHz}$. $\mathrm{C} 128$ had the tip of the cryoprobe placed on the apex of the bony wall of the cochlea for 2 applications of $1.5 \mathrm{~min}$ each, with an inter-application interval of $3 \mathrm{~min}$. Points with downward-pointing arrows represent frequencies where thresholds could not be obtained because equipment limitations prevented presenting higher sound levels. 


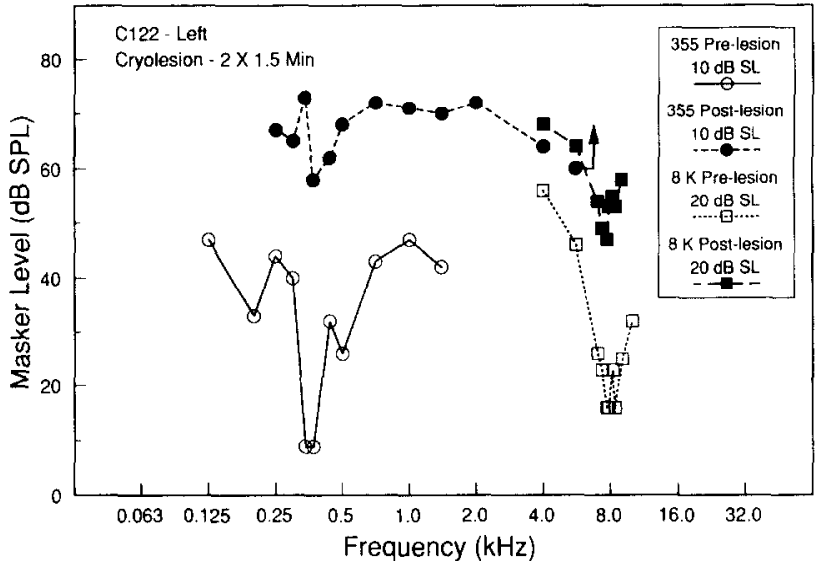

Fig. 5. This figure shows simultaneous-masked psychophysical tuning curves from the experimental ear of $\mathrm{C} 122$. Each tuning curve depicts the maximum level of the masker associated with detection of the test tone on $50 \%$ of the trials as a function of masker frequency. Tuning curves were determined with the test tone set to $0.355 \mathrm{kHz}$, $10 \mathrm{~dB}$ SL pre-lesion (open circles), and post-lesion (closed circles), and with the test tone set to $8.0 \mathrm{kHz}, 20 \mathrm{~dB}$ SL pre-lesion (open squares) and post-lesion (closed squares). The point with the upward-pointing arrow at $5.6 \mathrm{kHz}$ represents the maximum sound level that could be obtained, and is not a true threshold.

and no outer hair cells in this basilar membrane region. While portions of the apical cochlea of C130 could not be visualized nor the conditions of those cells assessed, that portion of C130's cochlea assumed to encode $0.355 \mathrm{kHz}$ had only a small percentage of hair cells present, similar to the cochleae of $\mathrm{C} 122$ and $\mathrm{C} 134$. The low-frequency post-lesion PTCs of the 3 subjects from which these data were obtained had extended highfrequency tails that showed increased masker effective-

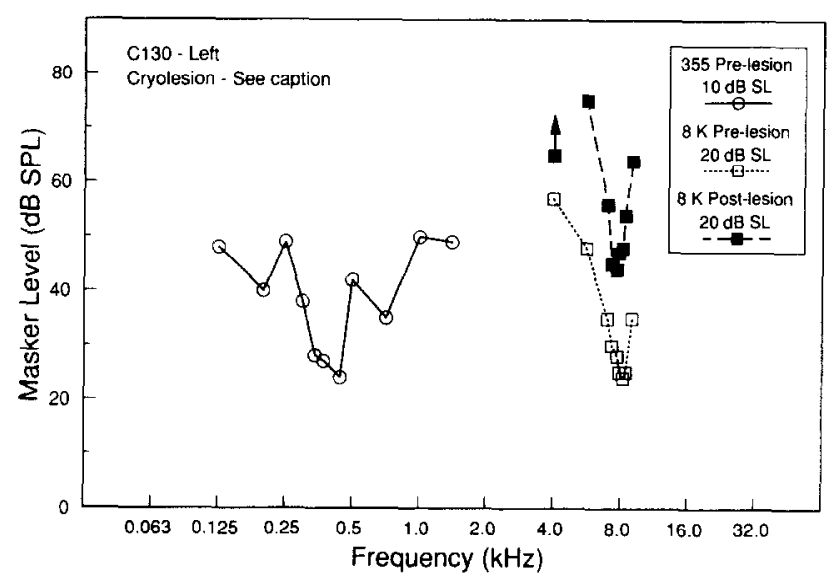

Fig. 6. This figure shows simultaneous-masked psychophysical tuning curves from the experimental ear of $\mathrm{C} 130$. Each tuning curve depicts the maximum level of the masker associated with detection of the test tone on $50 \%$ of the trials as a function of masker frequency. Tuning curves were determined with the test tone set to $0.355 \mathrm{kHz}$, $10 \mathrm{~dB}$ SL pre-lesion (open circles), and with the test tone set to 8.0 $\mathrm{kHz}, 20 \mathrm{~dB}$ SL pre-lesion (open squares) and post-lesion (closed squares). The point with the upward-pointing arrow at $4 \mathrm{kHz}$ represents the maximum sound level that could be obtained, and is not a true threshold.

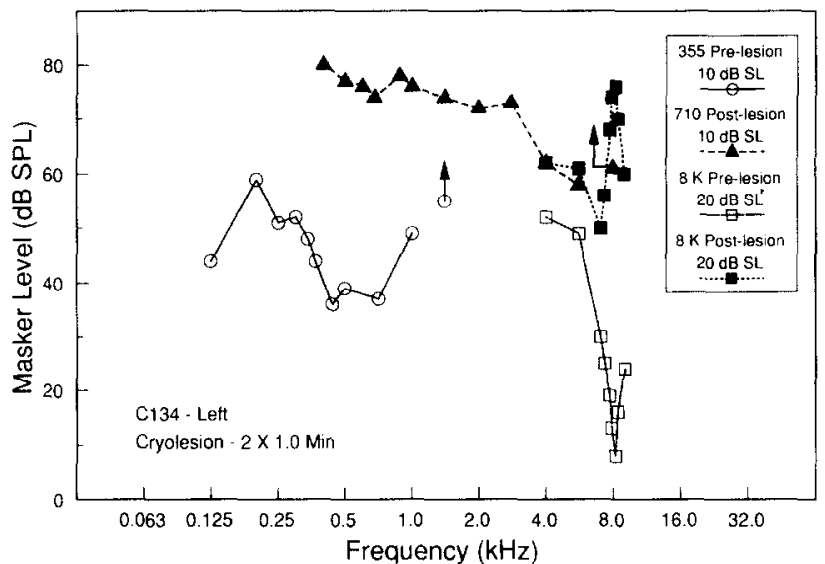

Fig. 7. This figure shows simultaneous-masked psychophysical tuning curves from the experimental ear of $\mathrm{C} 134$. Each tuning curve depicts the maximum level of the masker associated with detection of the test tone on $50 \%$ of the trials as a function of masker frequency. Tuning curves were determined with the test tone set to $0.355 \mathrm{kHz}$. $10 \mathrm{~dB}$ SL pre-lesion (open circles), with the test tone set to $0.71 \mathrm{kHz}$, $10 \mathrm{~dB} \mathrm{SL}$ post-lesion (closed triangles), and with the test tone set to $8.0 \mathrm{kHz}, 20 \mathrm{~dB}$ SL pre-lesion (open squares) and post-lesion (closed squares). The points with the upward-pointing arrows represent the maximum masker levels that were available at the time of testing, and are not true thresholds. Between the time the $0.355 \mathrm{kHz}$ pre-lesion function was obtained and the time the $0.71-\mathrm{kHz}$ function was obtained, the equipment was modified to allow higher stimulus levels.

ness with increases in masker frequency. These data suggest that basal cochlear locations normally responsive to high frequencies were responsible for detecting the low-frequency PTC test tone.

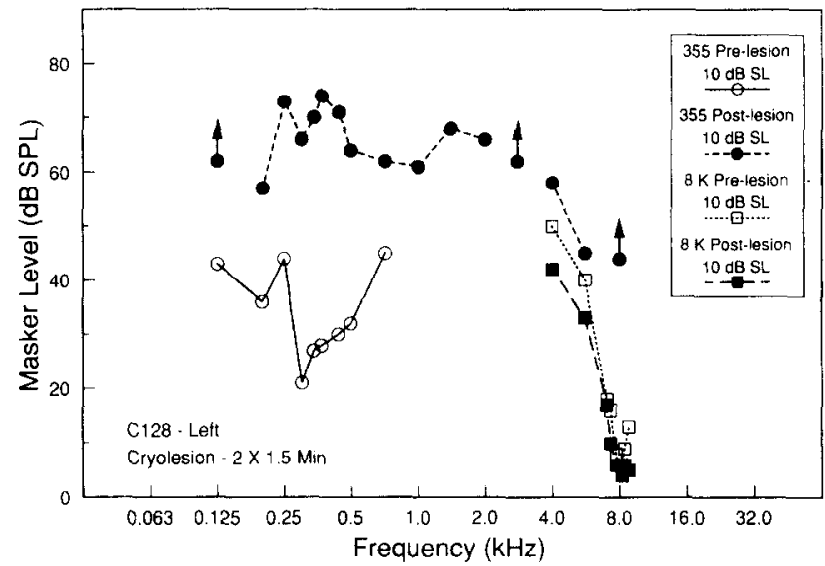

Fig. 8. This figure shows simultaneous-masked psychophysical tuning curves from the experimental ear of $\mathrm{C} 128$. Each tuning curve depicts the maximum level of the masker associated with detection of the test tone on $50 \%$ of the trials as a function of masker frequency. Tuning curves were determined with the test tone set to $0.355 \mathrm{kHz}$, $10 \mathrm{~dB}$ SL pre-lesion (open circles) and post-lesion (closed circles), and with the test tone set to $8.0 \mathrm{kHz}, 10 \mathrm{~dB}$ SL pre-lesion (open squares) and post-lesion (closed squares). 'The points with the upward-pointing arrows on the $0.355-\mathrm{kHz}$ function represent the maximum levels that were available from the equipment during testing and are not true thresholds. 
In contrast to these low-frequency data, the preand post-lesion high-frequency PTC functions from all 4 experimental subjects had nearly the same minima. Corresponding to the behavioral data, all inner and outer hair cells were preserved in C130 and C134 in the basilar membrane region presumed to encode 8.0 $\mathrm{kHz}$; for $\mathrm{C122}$, no outer hair cells remained in that region. Post-lesion high-frequency PTC functions from 3 of the 4 experimental subjects were elevated in level compared to the pre-lesion functions because these data were gathered at a higher SPL. Post-lesion data were determined at a higher SPL, but the same SL, in these subjects because each of these subjects had a $20-40 \mathrm{~dB}$ hearing loss at $8.0 \mathrm{kHz}$. These data suggest that pre- and post-lesion high-frequency tuning was mediated by the same basilar membrane location. The fact that the frequency of the minima of the highfrequency PTCs did not change further suggests that the low-frequency shift occurred because of the nearly complete apical hair cell destruction rather than because of an artifact related to a deterioration in behavioral performance.

\section{Frequency difference limens}

$\Delta \mathrm{F}$ thresholds at $355 \mathrm{~Hz}$ from $\mathrm{C} 125$, the control chinchilla, and from two of the experimental subjects are seen in Fig. 9. The control data indicate that the normal chinchilla detected a $20-25 \mathrm{~Hz}$ frequency difference at $355 \mathrm{~Hz}$, both in the absence and the presence of the high-pass masker. In contrast, the experimental subjects needed a larger frequency increment to detect a change in frequency at $355 \mathrm{~Hz}$. In the presence of the high-pass masker, this increment increased even more, from $58 \mathrm{~Hz}$ to $134 \mathrm{~Hz}$ for $\mathrm{C} 128$,

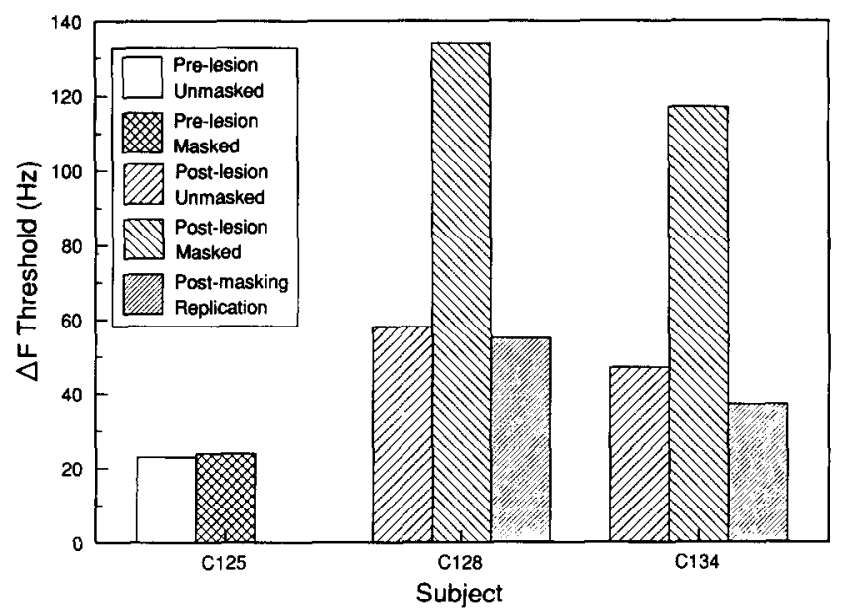

Fig. 9. Frequency difference limens at $355 \mathrm{~Hz}$ for $\mathrm{C} 125$, a control subject, and $\mathrm{C} 128$ and $\mathrm{C} 134$, two experimental subjects. All data from the experimental subjects were collected post-cryosurgery. The masker was a high-pass wide-band noise with a low-frequency cutoff of $0.71 \mathrm{kHz}$. The order of data acquisition was unmasked, masked, and replication. and from $47 \mathrm{~Hz}$ to $117 \mathrm{~Hz}$ for $\mathrm{C} 134$. For both of the experimental subjects, the $\Delta \mathrm{F}$ returned to its pre-masking magnitude after the masker was removed from the testing environment, as seen in Fig. 9. These data demonstrate that frequency discrimination at a low frequency was impaired but not obliterated following complete apical hair cell destruction. They also suggest that the remaining frequency discrimination ability was mediated by basally-located cells, since the $\Delta \mathrm{F}$ size increased in the presence of the masker.

\section{Discussion}

Hearing loss, cytocochleograms, and post-lesion masking data

The absolute threshold shift data from the 4 experimental subjects are consistent with the data from the Group 3 subjects described in an earlier report (Prosen et al., 1990b). In those animals, with complete or nearly complete apical cell loss and partial basal cell loss, low-frequency thresholds increased by $40-60 \mathrm{~dB}$. The hearing loss corresponding to complete apical-cell loss differs from the hearing loss corresponding to complete basal-cell loss, as reported by Hawkins et al. (1977); with complete basal-cell loss, high-frequency hearing is totally absent. The hearing that remains with complete apical hair cell loss suggests that redundant encoding mechanisms exist for low-frequency stimuli. While the place principle of hearing assumes that apical cells are responsible for low-frequency sensitivity, physiological data have shown that auditory nerve fibers with a higher characteristic frequency respond to lower frequencies, as is evidenced in the tails of auditory nerve tuning curves. Kiang and Moxon (1974), for example, reported that higher-frequency fibers can be driven in a time-locked fashion by low-frequency signals. As Long and Cullen (1988) have noted, one advantage of studying auditory perception in listeners with restricted low-frequency sensorineural hearing loss is that it permits the experimenter to look at the responses of the base of the cochlea, relatively uncontaminated by responses to unintentional apical stimulation. This feature permits comparisons between the current behavioral data and physiological findings like those of Kiang and Moxon.

In failing to show elevation in low-frequency thresholds, the masking data from the four experimental subjects suggest that the remaining low-frequency absolute sensitivity was not mediated by receptor cells located within the passband of the masker. As seen in the histopathological data, a small percentage of inner hair cells remained in the apical cochlea and these receptors may have been responsible for the remaining low-frequency sensitivity. This possibility is consistent 
with the findings of Clark and Bohne (1986) and Prosen et al. (1990b) that low-frequency stimuli can be detected at nearly normal levels in the presence of considerable apical receptor cell destruction.

Two alternative explanations exist, however, for the remaining low-frequency sensitivity. As noted earlier, in the pre-lesion masked testing environment, the masker did not elevate thresholds at $32.0 \mathrm{kHz}$. The first alternative explanation assumes that these veryhigh-frequency cochlear regions were responsible for the remaining low-frequency sensitivity. The second alternative assumes that two populations of receptors remained in the mid-frequency regions. The first population was sharply tuned with normal or slightly elevated tips and normal low-frequency tails. The second population had extremely elevated tips, and hypersensitive tails. Such high-threshold fibers have been described in normal ears by Liberman (1978) and in damaged ears by Liberman and Dodds (1984). In the threshold task with high-frequency stimuli, the population with relatively normal tuning curve tips and tails mediated the detection of the stimuli and it was these receptors that were affected by the masking. When detecting low-frequency stimuli, however, the hypersensitive tails of the second population of receptors were activated. Although these receptors had their best frequencics where the masker was effective, they were not themselves affected by the masker because of their high-threshold tips.

The noise masking data from $\mathrm{C} 128$ may bear on this second alternative. Before lesioning, the $52-\mathrm{dB}$ masker elevated low-frequency thresholds by $5-10 \mathrm{~dB}$, but did not produce a similar low-frequency shift after lesioning. This finding suggests that the low-frequency receptors which, before lesioning, had been directly affected by the $52-\mathrm{dB}$ masker were eliminated by the lesioning procedure, and that mid-frequency receptors, with hypersensitive low-frequency tails and elevated or missing tips, existed post lesion. These receptors were unaffected by the 52-dB masker, and mediated the detection of low-frequency signals.

\section{Psychophysical tuning curces}

Data from the 3 experimental subjects from which post-lesion low-frequency PTCs were obtained demonstrated a gradually decreasing threshold as masker frequency increased. This upward shift in masker effectiveness was very large - from 0.355 to $5.6 \mathrm{kHz}$ or more. It is possible that the shift was even more dramatic in magnitude; we could not use masker frequencies greater than $8.0 \mathrm{kHz}$ because the response of the speaker fell off above that frequency. These data are consistent with data from the human literature (Thornton and Abbas, 1980; Florentine and Houtsma. 1983; Goldstein et al., 1983; Tyler et al., 1983; Long and Cullen, 1988), suggesting that with a low-frequency sensorineural hearing loss, the detection of the test tone occurred at a 'high-frequency' place in the cochlea. These low-frequency PTC data also are consistent with the second alternative explanation of the noise masking data discussed above; namely, that low-frequency detection post lesion was mediated by basal receptors with hypersensitive tails and elevated or missing tips. The high-frequency PTC data, which remained relatively unchanged post lesion, also are consistent with this hypothesis since high-frequency test tones would be detected by the population of receptors that retained their low-threshold, sharply tuned tips.

Hence, in response to one of the experimental objectives stated in the introduction, basal receptors can mediate detection of low-frequency stimuli, but selectivity, as demonstrated in the PTC functions, differs from that carried out by the apical cochlea. The perceptual sequelae of this change were not explored in the present experiment, but might have important ramifications for speech perception, as suggested by Tyler et al. (1983).

Other reports have indicated that PTCs broaden with increasing level even in normal listeners (Small, 1959; Zwicker, 1974; Green et al., 1981; Nelson and Freyman, 1984; Smith et al., 1987b). Hence, broadened PTCs in the present experiment would be predicted because the post-lesion low-frequency PTCs were assessed at much higher levels than the pre-lesion lowfrequency PTCs. However, none of the studies cited above suggested that the minima of the function shifted to a higher frequency with increased level, as was found in the present experiment.

In agreement with the data from the present experiment, Florentine and Houtsma (1983) reported that, in one listener with a unilateral low-frequency hearing loss, the minima of low-frequency PTCs were increased in frequency. With increasing level, these minima decreased somewhat in frequency, although they still remained at higher frequencies than were found in this listener's normal ear. These data suggested that at higher PTC levels, undamaged apical and/or mid-turn fibers were recruited. We did not conduct our PTC experiments at more than one level because of the extent of the low-frequency hearing loss, and because the normal absolute thresholds at the low frequencies were high. In the future, it would be informative to examine PTC functions at multiple levels in subjects with less substantial apical receptor cell destruction.

\section{Frequency difference limens}

The $23-\mathrm{Hz} \Delta \mathrm{F}$ from the control subject at $355 \mathrm{~Hz}$ is within normal limits for the chinchilla; normal chinchilla $\Delta \mathrm{Fs}$ previously reported in the literature range from $12-15 \mathrm{~Hz}$ at $250-500 \mathrm{~Hz}$ (Nelson and Kiester, 
1978; Long and Clark, 1984) to $40-80 \mathrm{~Hz}$ at $1000 \mathrm{~Hz}$ (Prosen et al., 1989a). A comparison of the post-lesion $\Delta \mathrm{Fs}$ from the experimental subjects with the $\Delta \mathrm{F}$ from the control subject suggest that a 40-50 dB lowfrequency absolute threshold shift is accompanied by reduced frequency discrimination ability at low frequencies. However, to discount the possibility that this reduction merely reflected individual differences in $\Delta \mathrm{F}$ acuity, which may be substantial (see Prosen et al., 1990a), the masking experiment was carried out. The masking data from the frequency discrimination experiment suggest that the experimental subjects used midor basal-turn receptors to mediate frequency discrimination post-lesion, since the $\Delta \mathrm{Fs}$ increased in the presence of the high-pass masker. In contrast, masking data from the control subject suggest that the normal subject used apical cells to mediate the low-frequency discrimination, since $\Delta \mathrm{F}$ did not increase in the presence of the high-pass masker. Nelson and Stanton (1982) reached a similar conclusion while measuring low-frequency difference limens with and without a high-pass masker in a normal hearing human listener. In sum, the masking data from the $\Delta \mathrm{F}$ experiment strongly suggest that the lesioned subjects used a different cochlear region to discriminate between lowfrequency stimuli than did the normal control subject.

Frequency discrimination data from non-human and human subjects with a low-frequency sensorineural hearing loss suggest that, for a given degree of hearing loss, the impairment in frequency discrimination ability is greater at low-frequencies than at high-frequencies (Zurek and Formby, 1981; Clark and Bohne, 1986; Prosen et al., 1989b). Dissenting data were reported by Tyler et al. (1983), who noted that there was a poor correlation between compromised frequency discrimination ability at a low frequency and elevated pure tone thresholds at the same frequency. Reports of pitch matching data from listeners with low-frequency hearing losses also may be relevant to data on changes in frequency discrimination. While Butler and Albrite (1956) and Turner et al. (1983) reported that pitch matches were nearly normal in low-frequency impaired listeners, Florentine and Houtsma (1983) suggested that pitch matching ability changed when low-frequency thresholds were elevated. These conflicting data may be a function of the fact that not all low-frequency hearing losses have the same origin; some may reflect partial apical loss, while others reflect complete apical and partial basal loss.

Based on a comparison of the frcquency discrimination abilities of a variety of mammals, Prosen et al. (1990a) recently argued that frequency discrimination in the mammalian cochlea is conducted tonotopically. However, while normal listeners may use place information to discriminate frequency, listeners with cochlear pathology who receive unreliable place infor- mation may utilize periodicity information instead. 'This hypothesis has been advanced by Florentine and Houtsma (1983) and Turner et al. (1983). The fact that high-characteristic-frequency fibers have a time-locked response to the phase of low-frequency stimuli (Kiang and Moxon, 1974) indicates that these fibers can carry temporal information about low-frequency stimuli. Several reports have suggested that frequency discrimination is conducted by temporal processes for frequencies less than $4 \mathrm{kHz}$ (Srulovicz and Goldstein, 1983; Wakefield and Nelson, 1985). In the present study, the high-pass masker, while not elevating low-frequency absolute thresholds, may have increased the variance of the temporal firing patterns of the receptors detecting low frequencies post lesion. Such increases in the variance of PST histograms in the presence of a noise background have been demonstrated by Kiang et al. (1965). The increase in variance in time of firing would be expected to provide less reliable information on the frequency of the test stimulus, resulting in elevated $\Delta$ Fs.

Finally, data from this study support the hypothesis that frequency discrimination and frequency resolution, as reflected by PTC data, reflect different processes in the cochlea, as others have suggested (Tyler et al., 1983; Horst, 1987). That is, while frequency resolution is most likely a purely spectral process, frequency discrimination at low frequencies may be spectrally or temporally mediated.

We suggest using a conservative approach when interpreting the data from this experiment. While multiple psychophysical measures and histopathologic data were obtained from some of the same behavioral animals, not all types of data were collected from all experimental subjects, suggesting that the correlative conclusions be regarded as preliminary. Further, the cochlear mechanics from these severely damaged ears may be dramatically different from those in the normal ear. The direct effects of this alteration on frequency tuning and discrimination are unknown.

The data from this experiment are consistent with the hypothesis that, after apical hair cell destruction, mid- to high-frequency receptors with altered tuning are capable of mediating both the detection and discrimination of low-frequency stimuli. Future experiments in which the frequency resolving and discriminative powers of the cochlea are assessed after more limited apical hair cell destruction will delineate more clearly if normal demonstration of those powers is dependent on a full, or a partial, complement of apical hair cells.

\section{Conclusions}

- A small percentage of apical hair cells, highthreshold middle-turn cells, or cells located in the 
extreme base of the cochlea are sufficient to detect low-frequency stimuli with a $30-50 \mathrm{~dB}$ threshold shift.

- Psychophysical tuning curve data suggest that detection of low frequencies following apical-receptor-cell destruction may be mediated by receptors in higherfrequency regions of the cochlea.

- The low-frequency discrimination ability of listeners remains, but is compromised, following apical receptor cell destruction, suggesting that while this ability may be mediated by spectral processes in the normal ear, it can be carried out by temporal processes in the damaged ear.

\section{Acknowledgements}

We thank Ms. Nadine Brown who monauralized and cryolesioned all of the subjects in this paper and who performed the histological analysis of the cochlea from C134. We also thank Dr. Richard Altschuler, Dr. Yehoash Raphael, and the staff of their laboratory for histological analyses of the remaining cochleae. This research was supported by NIDCD Grant R01 DC00442 and by an equipment grant from the Office of the Vice President for Research of the University of Michigan.

\section{References}

Brown, J.N. and Nuttall, A.L. (1987) Cryoprobe-induced apical lesions in the chinchilla. I. Morphological effects of lesioning parameters. Hear. Res. 26, 301-309.

Butler, R.A. and Albrite, J.P. (1956) The pitch-discriminative function of the pathological ear. Arch. Otolaryngol, 56, 411-418.

Clark, W.W. and Bohne, B.A. (1986) Cochlear damage: Audiometric correlates? In: M.J. Collins, T.J. Glattke and L.A. Harper (Eds.), Sensorineural Hearing Loss: Mechanisms, Diagnosis, Treatment. Univ Iowa, Iowa City, pp. 59-82.

Eldredge, D.H., Miller, J.D. and Bohne, B.A. (1981) A frequencyposition map of the chinchilla cochlea. J. Acoust. Soc. Am. 69 , $1091-1095$

Florentine, M, and Houtsma, A.J.M. (1983) Tuning curves and pitch matches in a listener with a unilateral low-frequency hearing loss. J. Acoust. Soc. Am. 73, 961-965.

Goldstein, R., Karlovich, R.S., Tweed, T.S. and Kile, J.E. (1983) Psychoacoustic tuning curves and averaged electroencephalic responses in a patient with low-frequency sensory-neural hearing loss. J. Speech Hear. Disord. 48, 70-75.

Green, D.M., Shelton, B.R., Picardi, M.C. and Hafter, E.R. (1981) Psychophysical tuning curves independent of signal level. J. Acoust. Soc. Am. 69, 1758-1762.

Hawkins, J.E. Jr, Stebbins, W.C., Johnsson, L.G., Moody, D.B. and Muraski, A. (1977) The patas monkey as a model for dihydrostreptomycin ototoxicity. Acta Otolaryngol. (Stockh.) 83, 123-129.

Horst. J.W. (1987) Frequency discrimination of complex signals, frequency sclectivity, and specch perecption of hcaring-impaired subjects. J. Acoust. Soc. Am. 82, 874-885.

Kiang. N.Y.S. and Moxon, E.C. (1974) Tails of tuning curves of auditory-nerve fibers. J. Acoust. Soc. Am. 55, 620-630.

Kiang, N.Y.S., Watanabe, T., Thomas, E.X. and Clark, L.F. (1965) Discharge patterns of single fibers in the cat's auditory nerve. Res. Monog. 35, M.I.T. Press, Cambridge, MA.
Klein, J.O. (1983) Epidemiology and natural history of otitis media. Pediatrics 71, 639-640

Liberman, M.C. (1978) Auditory-nerve responses from cats raised in a low-noise chamber. J. Acoust. Soc. Am. 63, 442-455.

Liberman, M.C. and Dodds, L.W. (1984) Single-neuron labeling and chronic cochlear pathology. III. Stereocilia damage and alter ations of threshold tuning curves. Hear. Res. 16, 55-74.

Long, G.R. and Clark, W.W. (1984) Detection of frequency and rate modulation by the chinchilla. J. Acoust. Soc. Am. 75, 1184-119().

Long, G.R. and Cullen, J.K. Jr. (1988) Measures of signal processing in persons with sensorineural hearing loss below $6 \mathrm{kHz}$. J. Speech Hear. Res. 31, 659-669.

Nelson, D.A. and Freyman, R.L. (1984) Broadened forward-masked tuning curves from intense masking tones: Delay-time and probe-level manipulations. J. Acoust. Soc. Am. 75, 1570-1577.

Nelson, D.A. and Kiester. T.E. (1978) Frequency discrimination in the chinchilla. J. Acoust. Soc. Am. 64, 114-126.

Nelson, D.A. and Stanton, M.E. (1982) Frequency discrimination at $1200 \mathrm{~Hz}$ in the presence of high-frequency masking noise. $\mathrm{J}$. Acoust. Soc. Am. 71, 660-664.

Prosen, C.A., Halpern, D.L. and Dallos, P. (1989a) Frequency difference limens in normal and sensorineural hearing impaired chinchillas. J. Acoust. Soc. Am. 85, 1302-1313.

Prosen, C.A., Xu, X., Moody, D.B. and Stebbins, W.C. (1989b) Frequency difference limens following sensorineural damage in the monkey: High vs. low frequencies. Abstr. Assoc. Res. Otolaryngol. 222

Prosen, C.A., Moody, D.B., Sommers, M.S. and Stebbins, W.C. (1990a) Frequency discrimination in the monkey. J. Acoust. Soc Am. 88, 2152-2158.

Prosen, C.A., Moody, D.B., Stebbins, W.C., Smith, D.W., Sommers, M.S., Brown, J.N., Altschuler. R.A. and Hawkins, I.E. Ir. (1990h) Apical hair cells and hearing. Hear. Res. 44, 179-194.

Small, A.M. (1959) Pure-tone masking. J. Acoust. Soc. Am. 31, $1619-1625$.

Smith, D.W., Brown, J.N., Moody, D.B., Stebbins, W.C. and Nuttall. A.L. (1987a) Cryoprobe-induced apical lesions in the chinchilla II. Effects on behavioral auditory thresholds. Hear. Res. 26. $311-317$.

Smith. D.W., Moody, D.B. and Stebbins, W.C. (1987b) The effects of changes in absolute measurement level on psychophysical tuning curves in quiet and noise in patas monkeys. J. Acoust. Soc. Am. $82,63-68$.

Smith, D.W., Moody, D.B., Stebbins, W.C. and Norat, M.A. (1987c) Effects of outer hair cell loss on the frequency selectivity of the patas monkey auditory system. Hear. Res. 29, 125-138.

Srulovicz, P. and Goldstein, J.L. (1983) A central spectrum model: A synthesis of auditory-nerve timing-and placc-cues in monaural communication. J. Acoust. Soc. Am. 73, 1266-1276.

Thornton, A.R. and Abbas, P.J. (1980) Low-frequency hearing loss: Perception of filtered speech, psychophysical tuning curves and masking. J. Acoust. Soc. Am. 67, 638-643.

Turner, C.W., Burns, E.M. and Nelson. D.A. (1983) Pure-tone pitch perception and low-frequency hearing loss. J. Acoust. Soc. Am $73,966-975$.

Turner, C.W. and Nelson, D.A. (1982) Frequency discrimination in regions of normal and impaired sensitivity. J. Speech Hear. Res. 25, 34-41.

Tyler, R.S., Wood, E.J. and Fernandes, M. (1983) Frequency resolution and discrimination of constant and dynamic tones in normal and hearing impaired listeners. J. Acoust. Soc. Am. 74, 1190-1199.

Wakefield, G.H. and Nelson, D.A. (1985) Extension of a temporal model of frequency discrimination: Intensity effects in normal and hearing-impaired listeners. J. Acoust. Soc. Am. 77, 613-619.

Zurek, P.M. and Formby, C. (1981) Frequency discriminability of hearing-impaired listeners. J. Speech Hear. Res. 46, 108-112.

Zwicker, E. (1974) On a psychophysical equivalent of tuning curves. In: E. Zwicker and E. Terhardt (Eds.), Facts and Models in Hearing, Springer-Verlag, New York, pp. 132-141. 